\title{
RESEARCH PAPER \\ FOREIGN BODIES OF THE UPPER DIGESTIVE TRACT IN KOMFO ANOKYE TEACHING HOSPITAL
}

\author{
J. Opoku-Buabeng \\ Department of Eye, Ear, Nose and Throat, \\ School of Medical Sciences, KNUST, Kumasi
}

\begin{abstract}
Foreign body impaction in the upper digestive tract poses as an emergency condition to the otorhinolaryngologist as it discomforts the patient's ability to swallow food and water. The main objective of the study was to investigate the incidence, the anatomical site of impaction, the nature of the foreign body, the management and the outcome of foreign body impaction in the upper digestive tract, in Komfo Anokye Teaching Hospital (KATH) between January 2007 and December 2012. This was a cross-sectional retrospective study of cases which was diagnosed and managed as foreign body in the upper digestive tract within this period. The data collected from the clinical records of 1,412 patients was analyzed by using simple proportion and the statistical calculation by SPSS 16.0. Analysis showed male preponderance (61.8\%), with an average age of $28.8 \pm 1.9$ (mean \pm standard deviation). The commonest foreign body was bone (fish/meat/ chicken) in (45.7\%), followed by coin in (29.6\%) and then dentures in (14.3\%), etc. Direct laryngoscopy was performed in (63.0\%), rigid esophagoscopy under general anaesthesia in (34.0\%) and direct clinical removal in (3.0\%). $95.8 \%$ were successfully removed, (1.5\%) were not successfully removed and were referred to the Cardio-Thoracic Unit (CTU). In (2.7\%) of the cases the foreign bodies were not visualized and were managed conservatively. Although foreign body impaction in the upper gastrointestinal tract (GIT) was among one of the commonest emergency in ORL practices it can be a major challenge to the otorhinolaryngologist and as such measures need to be taken to prevent such incident.
\end{abstract}

Keywords: Foreign body, Upper digestive tract, Coin, McIntosh laryngoscope, ChevalierJackson esophagoscope

\section{INTRODUCTION}

Foreign body impaction in the upper digestive tract is among one of the Ear, Nose and Throat (ENT) emergencies in all communities which needs early diagnosis and prompt management.
Even though morbidity and mortality due to foreign body ingestion are rare, they may cause serious anxiety and complications in the patients. The upper digestive tract refers to the proximal portion of the digestive tract (from the 
61

\section{Opoku-Buabeng}

mouth to the distal portion of the esophagus).

Foreign body ingestion may occur in normal people who might accidentally swallow the foreign object or it can also happen intentionally in challenged or mentally ill individuals (Heim and Maughan, 2007). Children account for $75-85 \%$ of patients who presented with foreign bodies in the upper digestive tract with a preponderance of age 18-48 months. Children typically ingest objects they picked up and place in their mouths, such as coins, buttons, marbles, crayons and similar items (Schunk et al., 1994). Fish, meat and chicken bones, dentures, etc. are the most common foreign body ingestions in adults (Wu et al., 2011), (Gausam et al., 1994), (Kumar et al., 2003).

van As et al., (2003) in a Children's hospital in Cape Town, South Africa reported of an incidence rate of $4.2 \%$. In their study $67.7 \%$ were between 1 and 4 years and the pharnyx was the commonest site of impaction (38.4\%) and the esophagus $(12.9 \%)$. The nature of the foreign bodies was fish bone $(30.7 \%)$, plastic bags (13.6\%) and coins $(55.7 \%)$.

In children with swallowed foreign bodies, the incidence in males and females was equal (Balci and Eren, 2004), (Nadir et al., 2001), (Hurtado et al., 2011). In adults, the incidence of accidentally swallowed foreign bodies was slightly higher in men than women.

Coins are by far the most common foreign body ingested by children (Wyllie, 2006), (Waltzman, 2006). In a similar study conducted by Nandi and Ong (1978), bone ingestion constituted $42.6 \%$ whereas coins were found in $39.1 \%$ of the cases studied.

Kitcher et al., (2007) reported that in Korle Bu Teaching Hospital (KBTH) Accra, foreign bodies in the upper gastrointestinal tract was one of the common causes of Ear, Nose and Throat (ENT) emergency admissions. In their study, they reported of coins being the commonest $(20.1 \%)$, fish bones $(7.5 \%)$, dentures $(3.3 \%)$, and other foreign bodies (3.6\%).
Impacted food boluses are rare in children and adults. It usually presents as dysphagia (that is difficulty in swallowing) beginning acutely whilst eating. In patients presenting with food bolus impaction, there is a higher incidence of underlying esophageal pathology (strictures, achalasia or esophageal motility disorder) as compared to other patients with other foreign bodies (Webb, 1995), (Lao et al., 2003). The management of foreign bodies in the upper digestive tract depends on the age of the patient, nature of foreign body, the site of impaction and more importantly the facilities (medical equipments) available in the centre.

These days, the management of foreign bodies is not standardized in various institutions due to innovating technology and advances in instrumentation. The management options include an observational (direct) removal in the clinic, direct laryngoscopy using the McIntosh laryngoscope and McGill forceps under general anesthesia (GA) and rigid esophagoscopy using the Chevalier-Jackson esophagoscope and an appropriate long forceps. Currently the flexible endoscopic removal with video-imaging is practiced in most advanced institutions (Hachimi-Idrissi et al., 1998), (Seo, 1999), (Wu et al., 2011).

The main objective of conducting this study was to identify the incidence rate and the anatomical areas where foreign bodies can easily get impacted within the gastrointestinal tract. Secondly, it was to identify the nature of the foreign body, the management modalities and their outcomes. Lastly, there is little research on the subject within the sub-region (West Africa) and hence the reason for conducting the study.

\section{MATERIALS AND METHOD}

This was a retrospective study of all consecutive patients diagnosed with foreign body in the upper digestive tract in the Ear, Nose and Throat (ENT) Department of Komfo Anokye Teaching Hospital from January, 2007 to December 2012 inclusive. Data or variables in- 
cluding age, sex, nature of foreign body swallowed, anatomical site of impaction, procedure of removal and its outcome were obtained from patient's medical records in the ENT Department for this study.

Komfo Anokye Teaching Hospital is a tertiary health institution located in Kumasi, Ghana. It has a bed capacity of 1,500 and a major referral hospital in the northern sector of Ghana. The ENT Department is one of the sectors in the hospital with a bed capacity of 34 . All consecutive patients who presented at the department and were diagnosed with foreign body in the upper digestive tract during that period were involved in the study. Patient's records with definitive diagnosis and management procedure in order were chosen whereas patient's records with incomplete clinical entries were excluded. The variables captured included the age, sex, confirmed diagnosis, management and its outcome from the patients' clinical records.

Data collected were analyzed using simple statistical analyses and the statistical packages for the social scientists (SPSS) 16.0 for Windows Product. Their mean age with the standard deviation, male-to-female ratio and percentages of the nature of foreign body, the site of impaction and the various modalities of management and its' outcome were determined. The patients underwent various procedures in removing the foreign bodies including direct removal in the clinic using a tongue depressor and an oral forceps. Others were direct laryngoscopy using the Mcintosh laryngoscope and an esophagoscopy using the Chevalier-Jackson rigid esophagoscope.
Ethical consent and approval was sought from the Committee on Human Research Publication and Ethics from the Kwame Nkrumah University of Science and Technology (KNUST) and Komfo Anokye Teaching Hospital (KATH).

\section{RESULTS}

In this study, 1,412 patients were diagnosed as cases of foreign body ingestion into the upper digestive tract among the 90,660 seen within the 5-year period (DEENT KATH, Performance Review, 2012). There was therefore an incidence rate of $1.6 \%$ of foreign body ingestion within this 5-year period of study.

The patients consisted of 872 males $(61.8 \%)$ and 540 females $(38.2 \%)$, with a male-tofemale ratio of 1,6: 1, as shown in Table 1.

The patients' ages ranged between 10 months and 78 years. Of all the patients involved in this study $26.3 \%$ were 0 -10years, $12.7 \%$ were 11 20years, $14.7 \%$ were 21-30years and $20.0 \%$ were $31-40$ years. With the remaining, $10.1 \%$ were between $41-50$ years, $8.2 \%$ were $51-60$ years, $6.3 \%$ were $61-70$ years and $1.7 \%$ were 71-80 years. The mean age of the patients was 28.8 years \pm 1.9 (Mean \pm standard deviation) as shown in Table 2.

In $2.6 \%$ of the patients, the foreign body was lodge in the tonsillar fossa, in $13.6 \%$ was lodged on the pharyngeal wall, in $20.5 \%$ was lodged in the piriform fossa whereas in $48.1 \%$ it was lodged in the cricopharyngeal sphincter, that's the upper esophageal sphincter (UES).

Table 1: Gender distribution

\begin{tabular}{lcc}
\hline Gender & Frequency & Percentage $\%$ \\
\hline Males & 872 & 61.8 \\
Females & 540 & 38.2 \\
Total & $\mathbf{1 4 1 2}$ & $\mathbf{1 0 0}$ \\
\hline
\end{tabular}

Male-to-female ratio 1.6: 1 
Table 2: Age distribution

\begin{tabular}{ccc}
\hline Age group (years) & Frequency & Percentage \% \\
\hline $0-10$ & 372 & 26.3 \\
$11-20$ & 179 & 12.7 \\
$21-30$ & 207 & 14.7 \\
$31-40$ & 282 & 20.0 \\
$41-50$ & 142 & 10.1 \\
$51-60$ & 116 & 8.2 \\
$61-70$ & 89 & 6.3 \\
$71-80$ & 25 & 1.7 \\
\hline
\end{tabular}

Mean age \pm standard deviation $(28.8 \pm 1.9)$ years

Table 3: Anatomical site of impaction

\begin{tabular}{lcc}
\hline Anatomical site in GIT & Frequency & Percentage \% \\
\hline Tonsillar fossa & 37 & 2.6 \\
Pharyngeal wall & 192 & 13.6 \\
Piriform fossa & 289 & 20.5 \\
Cricopharyngeal sphincter & 679 & 48.1 \\
Upper one-third esophagus & 75 & 5.3 \\
Mid one-third esophagus & 82 & 5.8 \\
Lower one-third esophagus & 58 & 4.1 \\
\hline
\end{tabular}

Again in 5.3\% it was localized in the upper one -third of the esophagus, in $5.8 \%$ it was in the mid one-third of esophagus whereas in $4.1 \%$ it was in the lower one-third of the esophagus, including the lower esophageal sphincter as shown in Table 3.

The most common foreign body was bone (fish, meat, chicken etc) in $45.7 \%$, then coin in $29.6 \%$, dentures in $14.3 \%$ and food bolus in $4.8 \%$. The rest were nail/office pins/safety pins, etc. in $2.6 \%$, metallic objects (eg. Crown cork, ear rings, disk batteries, parts of key holder, etc. in $1.7 \%$ and nonspecified objects (eg. Parts of toys, piece of pencil, medications, chewing stick, plastic pieces) etc. in $1.3 \%$ as shown in Table 4.
Direct laryngoscopy was performed (under GA) in $63.0 \%$, a rigid esophagoscopy (under GA) with the Chevalier-Jackson esophagoscope) in $34.0 \%$ and direct removal in clinic in $3.0 \%$ of the cases as shown in Table 5.

In $95.8 \%$ the foreign bodies were successfully removed whereas in $2.7 \%$ of the cases the foreign bodies were not visualized. The $1.5 \%$ foreign bodies which could not be removed turned out to be cases impacted in the upper airway and were therefore sent to the Cardio-Thoracic Unit (CTU - KBTH) for surgical removal by the cardiothoracic surgeon, as shown in Table 6. 
Table 4: Nature of foreign body

\begin{tabular}{lcc}
\hline Nature of FB & Frequency & Percentage\% \\
\hline Inorganic & & \\
Coin & 418 & 29.6 \\
Denture & 201 & 14.3 \\
Nail/office pins & 37 & 2.6 \\
Metallic (Crown cork, key holder, disk batteries, etc.) & 24 & 1.7 \\
Organic & & \\
Bone (Fish, meat, chicken) & 646 & 45.7 \\
Food bolus & 68 & 4.8 \\
Nonspecified & 18 & 1.3 \\
\hline
\end{tabular}

Table 5: Management modalities

\begin{tabular}{lcc}
\hline Mode of management & Frequency & Percentage\% \\
\hline Direct laryngoscopy & 889 & 63.0 \\
Rigid esophagoscopy & 481 & 34.0 \\
Direct removal (in clinic) & 42 & 3.0 \\
\hline
\end{tabular}

Table 6: Management outcome

\begin{tabular}{lcc}
\hline Outcome & Frequency & Percentage\% \\
\hline Successful removal & 1,353 & 95.8 \\
Non-successful removal & 21 & 1.5 \\
FB not visualized & 38 & 2.7 \\
\hline
\end{tabular}

\section{DISCUSSION}

Foreign body ingestion in the upper digestive tract is a common problem in the practice of otorrhinolaryngology. In this study the incidence rate of foreign body ingestion was $1.6 \%$ as compared to a paediatrician's perspective of 4.2\% (van As et al., 2003) in Cape Town, South Africa.

There was a male-to-female ratio of 1:6: 1 , whereas (Balci and Eren, 2004), (Nadir et al., 2001), (Hurtado et al., 2011) reported of an invariable difference in children but a slightly higher incidence in adults. The commonest site of impaction was the cricopharyngeal sphincter (48.1\%). (Willie, 2006) and (Waltzman, 2006) reported of about $75 \%$ in the cricopharyngeal sphincter in children and $70 \%$ in adults. This might be due to the fact that the cricopharyngeal sphincter is the narrowest of all 
the digestive tract sphincters. Bones including that of fish, meat and chicken were the commonest foreign body (45.7\%) especially in adults and coins $(29.6 \%)$ especially in children. This is in concordance to the findings of (Willie, 2006) and (Waltzman, 2006) who reported of higher incidence of coin as a foreign body ingested by children. This might be due to the fact that some parents often give coins to children to send to school and other outings thus making them more prone to swallowing of coins and secondly the cricopharyngeal inlet is small in children. Meanwhile Nandi and Ong, (1978) reported of the incidence of bone in $42.6 \%$ whereas coin was identified in $39.1 \%$ in a similar study. The low incidence of bone and coin ingestion may be due to the fact that in most advanced countries, boneless fishes and meats are often used in meals than within our environment. Again over there, they rarely chew bones while it frequently occurs here. Secondly, parents do not give coins to their children as it often happens here.

Direct laryngoscopy using the McIntosh laryngoscope and the McGill forceps under GA was performed in $63.0 \%$ of the patients as the mainstem management, $34.0 \%$ underwent a rigid esophagsoscopy using the Chevalier-Jackson esophagoscope with FB forceps and a direct removal in the clinic with long artery forceps in $3.0 \%$. Currently flexible endoscopy is one of the new techniques used in the removal of foreign bodies in the upper digestive tract. Gmeiner et al., (2007) reported that the advantages of flexible over rigid endoscopy was low rate severe complications, better patient comfort with a lower rate of dysphagia and lack of requirement for general anaesthesia.

Yalçin et al., (2007) performed a similar study in children in which laryngoscopy was performed in $12 \%$ of them, rigid esophagoscopy in $51 \%$ and flexible endoscopy in $5.0 \%$. Even though some literature have reported of the efficiencies with the flexible endoscopy, none was performed in this study because of lack of the instrument in the department.
In this study there was a success rate of removal of the foreign body in $95.8 \%$ of the cases. $1.5 \%$ of the foreign bodies was unsuccessful during the removal process whereas in $2.7 \%$ of the cases, the foreign bodies were not visualized. Yalçin et al., (2007) reported of a removal success rate of $68.0 \%$, and $7.1 \%$ unsuccessful. The unsuccessful cases were all referred to the Cardio-Thoracic Unit (KBTH) for surgical removal.

\section{CONCLUSION}

Foreign body impaction in the upper digestive tract is among the common emergencies in clinical practice of otorhinolaryngology and it is equally frequent in both children and adults. This condition may cause a lot of inconveniences to the patient based on the site of entrapment, the nature of foreign body and the type of management applied. The most common site of impaction is the cricopharyngeal sphincter and bones are the common identified foreign bodies. Most foreign bodies were removed without any complications.

It will be better to provide education on how to prevent such eventualities by creating public awareness through the media, in schools and in various health institutions within the country. Infants and young children should be kept under surveillance all the time and parents must be advised not to give coins to their children. Swallowing without proper chewing, i.e. eating in a hurry, talking whilst eating should be avoided. People with dentures should be more cautious as they are more likely to swallow bone, due to reduced sensitivity and inability to chew properly. Ill-fitting dentures must be properly fixed by competent dental technicians or technologist. Carpenters, dressmakers, office staff, etc. must avoid putting nails and other pins into their mouth whilst working. The public must also be made aware of the consequences and patients should be asked to consult the otorhinolaryngology professional first should such a situation occur. 
Foreign body impaction in the upper digestive tract ...

\section{REFERENCES}

Balci, A. E. and Eren, M. N. (2004). Esophageal foreign bodies under cricopharyngeal level in children: an analysis of 1116 cases. Interactive Cardiovascular Thoracic Surgery, 3(1): 14-18.

Gausam, V., Phillips, J., Bowmer, H. and Reichl, M. (1994). Foreign body in the throat. Journal of Accident and Emergency Medicine, 11(3): 113-115.

Gmeiner, D., von Rahden, B. H., Meco, C., Hutter, J., Oberascher, G. and Stein, H. J. (2007). Flexible versus rigid endoscopy for treatment of foreign body impaction in the esophagus. Surgical Endoscopy, 21(11): 2026-2029.

Hachimi-Idrissi, S., Come, L. and Vandenplas, Y. (1998). Management of ingested foreign bodies in childhood: Our experience and review of the literature. European Journal of Emergency Medicine, 5(3): 319-323.

Heim, S. W. and Maughan, K. L. (2007). Foreign bodies in the Ear, Nose and Throat. American Family Physician, 76(8): 11851189.

Hurtado, C. W., Furuta, G. T. and Kramer, R. E. (2011). Etiology of esophageal food impactions in children. Journal of Pediatric Gastroenterology and Nutrition, 52(1): 4346.

Kitcher, E. D., Jangu, A. and Baidoo, K. (2007). Emergency Ear, Nose and Throat Admissions at the Korle-Bu Teaching Hospital. Ghana Medical Journal, 41(1): 9-11.

Kumar, M., Joseph, G., Kumar, S. and Clayton, M. (2003). Fish bone as a foreign body. Journal of Laryngology and Otology, 117(7): 568 -569 .

Lao, J., Bostwick, H. E. and Berzin, S. (2003). Esophageal food impaction in children.
Pediatric Emergency Care, 19(6): 402-407.

Nadir, A., Sahin, E., Nadir, I., Karadayi, S. and Kaptanoglu, M. (2011). Esophageal foreign bodies: 177 cases. Diseases of Esophagus, 24 (1): 6-9.

Nandi, P. and Ong, G. B. (1978). Foreign body in the esophagus: Review of 2394 cases. British Journal of Surgery, 65(1): 5-9.

Schunk, J. E., Harrison, A. M., Cornelli, H. M. and Nixon, G. W. (1994). Fluoroscopic foley catheter removal of esophageal foreign bodies in children: experience with 415 episodes. Pediatrics, 94(5): 709-14.

Seo, J. K. (1999). Endoscopic management of gastrointestinal foreign bodies in children. Indian Journal of Pediatrics, 66(1): S75-80.

Tibbling, L., Bjorkhoel, A., Jansson, E. and Stenkvist, M. (1995). Effect of spasmolytic drugs on esophageal foreign bodies. Dysphagia, 10(2): 126.

van As, A. B., Du Toit, N., Wallis, L., Stool, D., Chen, X. and Rode, H. (2003). "The South African experience with ingestion injury in children". International Journal of Pediatric Otorhinolaryngology, 67(1): S175S178.

Waltzman, M. (2006). Management of esophageal coins. Pediatric Emergency Care, 22 (5): $367-373$.

Webb, W. A. (1995). Management of foreign bodies of the upper gastrointestinal tract: update. Gastrointestinal Endoscopy, 41(1): 39-51.

Wu, W. T., Chiu, C. T., Kuo, C. J., Lin, C. J., Chu, Y. Y., Tsou, Y. K. and Su, M. Y. (2011). Endoscopic management of suspected esophageal foreign body in adults. Diseases of Esophagus, 24(1): 131-137 . 
Wyllie, R. (2006). Foreign bodies in the gastrointestinal tract. Current Opinion Pediatrics, 18(5):563-564.

Yalçin, S., Karnak, I., Ciftci, A. O., Senocak,
M. E., Tanyel, F. C. and Büyükpamukçu, N. (2007). Foreign body ingestion in children: an analysis of pediatric surgical practice. Pediatric Surgical International, 23 (8): 755 761 